\title{
Using Digital Stories In Writing Instruction For Secondary Students With Disabilities
}

\author{
Joy Xin \\ Department of Language, Literacy and Special Education, Rowan University, New Jersey, USA \\ xin@rowan.edu
}

\begin{abstract}
This presentation will discuss two studies using computer-assisted instruction with digital stories to teach expressive writing to secondary students with disabilities. One study focused on students with autism, and the other involved those with learning disabilities. "PhotoStory" of Microsoft in the Windows platform was used to allow learners to post, crop, move and rotate digital pictures, typing texts, and recording voices, in order to produce a story in a slideshow format. As a result, students learned writing skills to develop their compositions to increase the length, correct words and sentences, and improve quality of writing.

Index Terms - Technology, Writing, Secondary Students,
\end{abstract} Disabilities

Writing is an important medium for students to communicate their thoughts, feelings, and beliefs (Graham \& Perin, 2007). With the development of new media using technology, writing is necessary in one's daily life, such as communicating with family members and friends via websites, e-mails, and social media via Facebook. However, writing performance of many adolescents is below the level of required proficiency. To improve adolescents' writing skills, writing has become an integral part of the middle school and high school's curriculum. Yet, writing is a difficult subject area or skill for students, especially for those with disabilities.

Although developing writing skills is a challenge for students with disabilities, the acquisition of these skills is important for them in a variety of contexts to express their idea and enhance their function in school. For example, they need written notes to make direct requests, engage in social conversations, create compositions to express their feelings, and to communicate with others. Thus, writing instruction is critical to students to learn writing and to improve writing skills.

According to Ganz, Earles-Vollrath and Cook (2011), visually based instruction, such as using computer programs to demonstrate visual images is effective more than traditional approaches for students with disabilities. It seems that a visually based approach assists these students to focus and maintain their attention (Simpson, Myles \& Ganz, 2008). Thus, their abilities to independently complete unfamiliar tasks are enhanced through visual cues (Ganz, Earles-Vollrath, \& Cook, 2011). The nature of visually based instruction should allow these students to review visual cues and to make abstract concepts concrete. Successful approaches to instruction therefore should include pictures and photos that present class activities. These visual displays should provide more concrete examples that will be especially beneficial to students with disabilities.
A story presented digitally on a computer is referred to as digital story telling. Such digital stories often include multimedia texts consisting of still images complemented by a narrated soundtrack that tells a story. Computer programs such as iMovie on Mac computers and MovieMaker on Windows of PC computers present user-friendly options to develop digital stories. An example that is easily accessible is found in "PhotoStory", a free application from Microsoft available for use in the Windows platform (http://www.microsoft.com/download/en/details.aspx?id=1113 2). This program allows users to post, crop, move and rotate digital pictures, typing texts, and recording voices, in order to produce a story in a slideshow format. Thus, users are able to add stunning special effects, soundtracks, and individual's own voice narration to their photo story, as well as titles and captions. Creating such digital stories serves students a motivator to engage in the class activity, and provides an alternative conduit of expression for those who struggle with writing traditional text materials (Sylvester \& Greenidge, 2009). Computer-assisted instruction with such digital images serves as a visual system to support students in brainstorming, expanding, and organizing ideas (Sylvester \& Greenidge, 2009). This application of a computer-technology as a learning medium presents materials in a visual system to benefit learners with disabilities in learning difficult subjects, such as writing (Pennington, Stenhoff, Gibson, \& Ballou, 2012).

The purpose of this presentation is to discuss the effects of this visual system created in teaching expressive writing to students with disabilities, especially those with learning disabilities and autism. Two recent studies using the digital stories assisting two groups of students, one group with learning disabilities, and another with autism in learning expressive writing will be reported to share with audience. Both studies examined the extent students with disabilities could develop their own digital stories to improve their writing skills in terms of total written words(TW), correct words(CW), complete sentences(CS), and general writing quality $(\mathrm{QW})$.

During the studies, a single subject research design of $\mathrm{ABC}$ phases (A: baseline, B: intervention, $\mathrm{C}$ : maintenance) was used to evaluate the students' performance. During the baseline (prior to the intervention), the students were given a topic with a picture prompted for free writing. A total of three stories were assigned in class to measure their narrative writing abilities to establish their performance levels. The total number of words written, correct words, complete sentences, 
and writing quality was recorded for each story. During the intervention, the computer-assisted instruction using digital stories was provided, and six stories were developed with one story in two weeks. Two weeks after the intervention, each participant was administrated two story writing task using the digital story program to measure their achievement levels. These stories were written without the teacher's modeling, but students developing their own stories assisted by the instructor if needed.

The results showed that the students made progress in their writing including an increase of total number of words in compositions, total number of complete sentences, and correct wording. In addition, the quality of their writing was improved compared to their written assignments prior to the instruction. All participating students were able to learn how to develop their own stories by posting their own pictures, finding pictures by searching in the internet and recording their voices to describe their stories. This learning by doing activity reinforced their learning and motivated them to expand and organize their ideas for writing. In addition, the voiced narratives in recording helped these students transfer their ideas into written text, which is one of the difficult tasks for students with disabilities.
This presentation will demonstrate the specific lessons delivered in writing instruction to help students with disabilities, as well as the results of the two studies. Meanwhile, using technology in writing instruction will be further explored to make it potential in classrooms to support students' learning.

\section{References}

[1] Graham, S., \& Perin, D. (2007). What we know, what we still need to know: Teaching adolescents to write, Scientific Studies of Reading, 11 (40), 313-335.

[2] Ganz, J., Earles-Vollrath, T, \& Cook, K. (2011), Video Modeling: A visually based intervention for children with autism spectrum disorder, Teaching Exceptional Children, 43(6), 8-19.

[3] Pennington, R. C., Stenhoff, D. M., Gibson, J., Ballou, K. (2012), Using simultaneous prompting to teach computer-based story writing to a student with autism, Education and Treatment of Children, 35 (3), 389406.

[4] Simpson, R. L, Myles, B. S., \& Ganz, J. B. (2008). Efficacious interventions and treatments for learners with autism spectrum disorders. In R. L.

[5] Simpson \& B. S. Myles (Eds.) Educating children and youth with autism: Strategies for effective practice ( $2^{\text {nd }}$ ed., pp 477-512). Austin, TX:PRO-ED.

[6] Sylvester, R., \& Greenidge, W. (2009), Digital storytelling: Extending the potential for struggling writers. The Reading Teacher, 63(4), 284- 295 